\title{
FIXED POINT INDICES AND MANIFOLDS WITH COLLARS
}

\author{
CHEN-FARNG BENJAMIN AND DANIEL HENRY GOTTLIEB
}

Received 7 December 2004; Revised 25 April 2005; Accepted 24 July 2005

This paper concerns a formula which relates the Lefschetz number $L(f)$ for a map $f$ : $M \rightarrow M^{\prime}$ to the fixed point index $I(f)$ summed with the fixed point index of a derived map on part of the boundary of $\partial M$. Here $M$ is a compact manifold and $M^{\prime}$ is $M$ with a collar attached.

Copyright (c) 2006 C.-F. Benjamin and D. H. Gottlieb. This is an open access article distributed under the Creative Commons Attribution License, which permits unrestricted use, distribution, and reproduction in any medium, provided the original work is properly cited.

\section{Introduction}

This paper represents the first third of a Ph.D. thesis [2] written by the first author under the direction of the second author at Purdue University in 1990. The thesis is entitled "Fixed Point Indices and Transfers, and Path Fields" and it contains, in addition to the contents of this manuscript, a formula analogous to (1.1), which relates to Dold's fixed point transfers and a study of path fields of differential manifolds in order to relate the formula in this manuscript with an analogous formula involving indices of vector fields. These results are related to the papers $[1,3,4,7,8,14,16]$

Let $M$ be a compact differentiable manifold with or without boundary $\partial M$. Assume $V$ is a vector field on $M$ with only isolated zeros. If $M$ is with boundary $\partial M$ and $V$ points outward at all boundary points, then the index of the vector field $V$ equals Euler characteristic of the manifold $M$. This is the classical Poincaré-Hopf index theorem. (A 2dimensional version of this theorem was proven by Poincare in 1885; in full generality the theorem was proven by Hopf [13] in 1927). In particular, the index is a topological invariant of $M$; it does not depend on the particular choice of a vector field on $M$.

Morse [15] extended this result to vector fields under more general boundary conditions, namely, to any vector field without zeros on the boundary $\partial M$; he discovered the following formula:

$$
\operatorname{Ind}(V)+\operatorname{Ind}\left(\partial_{-} V\right)=\chi(M)
$$

Hindawi Publishing Corporation

Fixed Point Theory and Applications

Volume 2006, Article ID 87657, Pages 1-8

DOI 10.1155/FPTA/2006/87657 
where $\chi(M)$ denotes the Euler characteristic of $M$ and $\partial_{-} V$ is defined as follows. Let $\partial_{-} M$ be the open subset of the boundary $\partial M$ containing all the points $m$ for which the vectors $V(m)$ point inward, and let $\partial V$ be the vector field on the boundary $\partial M$ obtained by first restricting $V$ to the boundary and then projecting $\left.V\right|_{\partial M}$ to its component field tangent to the boundary. Then $\partial_{-} V=\left.\partial V\right|_{\partial_{-}}$. Furthermore, in the same paper, Morse generalized his result to indices of vector fields with nonisolated zeros. This is the formula (1.1). Now (1.1) was rediscovered by Gottlieb [10] and Pugh [17]. Gottlieb further found further interesting applications in $[9,11,12]$. Throughout this paper, we will call formula $(1.1)$ the Morse formula for indices of vector fields.

We consider maps $f: M \rightarrow M^{\prime}$ from a compact topological manifold $M$ to $M^{\prime}$, where $M^{\prime}$ is obtained by attaching a collar $\partial M \times[0,1]$ to $M$. If $f$ has no fixed points on the boundary $\partial M$, we prove Theorem 3.1 which is the fixed point version of the Morse formula:

$$
I(f)+I\left(\left.r \circ f\right|_{\partial \_}\right)=L(r \circ f)
$$

where $I$ denotes the fixed point index, $r$ is a retraction of $M^{\prime}$ onto $M$ which maps the collar $\partial M \times I$ onto the boundary $\partial M, \partial \_M$ is an open subset of $\partial M$ containing all the points $x \in \partial M$ mapped outside of $M$ under $f$, and $L(r \circ f)$ is the Lefschetz number of the composite map $r \circ f$.

In particular, if the map $r \circ f$ is homotopic to the identity map, we have

$$
I(f)+I\left(\left.r \circ f\right|_{\partial_{-} M}\right)=\chi(M)
$$

which is similar to the Morse formula; and the map $r \circ f \mid \partial_{-} M$ is analogous to the vector field $\partial_{-} V$.

Formula (1.3) was independently obtained by A. Dold (private letter to D. Gottlieb).

This paper is organized as follows: in Section 2, we list some properties of fixed point indices; our first main result, Theorem 3.1, is proven in Section 3.

\section{Fixed point index and its properties}

In this section, we use the definition of fixed point index and some well-known results on fixed point index given by Dold in [5] or [6, Chapter 7] to obtain an equation for fixed point indices (Theorem 3.1) analogous to the Morse equation for vector field indices described in the introduction.

Let $X$ be an Euclidean neighborhood retract $(E N R)$. Consider maps $f$ from an open subset $V$ of $X$ into $X$ whose fixed point set $F(f)=\{x \in V \mid f(x)=x\}$ is compact. Dold [5] defined the fixed point index $I(f)$ and proved the following properties.

Localization 2.1. Let $f: V \rightarrow X$ be a map such that $F(f)$ is compact, then $I(f)=I\left(\left.f\right|_{W}\right)$ for any open neighborhood $W$ of $F(f)$ in $V$. 
Additivity 2.2. Given a map $f: V \rightarrow X$ and $V$ is a union of open subsets $V_{j}, j=1,2, \ldots, n$, such that the fixed point sets $F^{j}=F(f) \cap V_{j}$, are mutually disjoint. Then for each $j$, $I\left(\left.f\right|_{V_{j}}\right)$ is defined and

$$
I(f)=\sum_{j=1}^{n} I\left(\left.f\right|_{V_{j}}\right) .
$$

Units 2.3. Let $f: V \rightarrow X$ be a constant map. Then

$$
\begin{array}{ll}
I(f)=1 & \text { if } f(V)=p \in V, \\
I(f)=0 & \text { if } f(V)=p \notin V .
\end{array}
$$

Normalization 2.4. If $f$ is a map from a compact $E N R X$ to itself, then $I(f)=L(f)$, where $L(f)$ is the Lefschetz number of the map $f$.

Multiplicativity 2.5. Let $f: V \rightarrow X$ and $f^{\prime}: V^{\prime} \rightarrow X^{\prime}$ be maps such that the fixed point sets $F(f)$ and $F\left(f^{\prime}\right)$ are compact, then fixed point index of the product $f \times f^{\prime}: V \times V^{\prime} \rightarrow$ $X \times X^{\prime}$ is defined and

$$
I\left(f \times f^{\prime}\right)=I(f) \cdot I\left(f^{\prime}\right) .
$$

Commutativity Axiom 2.6. If $f: U \rightarrow X^{\prime}$ and $g: U^{\prime} \rightarrow X$ are maps where $U \subseteq X$ and $U^{\prime} \subseteq X^{\prime}$ are open subsets, then the two composites $g f: V=f^{-1}\left(U^{\prime}\right) \rightarrow X$ and $f g: V^{\prime}=$ $g^{-1}(U) \rightarrow X^{\prime}$ have homeomorphic fixed point sets. In particular, $I(f g)$ is defined if and only if $I(g f)$ is defined, in that case,

$$
I(f g)=I(g f)
$$

Homotopy Invariance 2.7. Let $H: V \times I \rightarrow X$ be a homotopy between the maps $f_{0}$ and $f_{1}$. Assume the set $F=\{x \in V \mid H(x, t)=x$ for some $t\}$ is compact, then

$$
I\left(f_{0}\right)=I\left(f_{1}\right) .
$$

For our purposes, it is useful to reformulate the properties of Additivity 2.2 and Homotopy Invariance 2.7 in the form of the following propositions. These reformulations are found in Brown's book [4], and they form part of an axiom system for the fixed point index. The five axioms are a subset of Dold's properties. They consist of localization, homotopy invariance, addititvity, normalization, and commutivity. We will show that the main formula will follow from these axioms. We will give an alternate proof in Section 3.

Proposition 2.8. Assume $X$ is compact and $V$ is an open subset of $X$. Let $f: \bar{V} \rightarrow X$ be a map without fixed points on $B d(V)$. If $\left\{V_{j}\right\}, j=1,2, \ldots, n$ are mutually disjoint open subsets of $V$ and whose union $\bigcup_{j=1}^{n} V_{j}$ contains all the fixed points of $f$, then

$$
I\left(\left.f\right|_{V}\right)=\sum_{j=1}^{n} I\left(\left.f\right|_{V_{j}}\right) .
$$


Proposition 2.9. Assume $X$ is compact and $V$ is an open subset of $X$. Let $\bar{H}: \bar{V} \times I \rightarrow X$ be a homotopy from $\bar{f}_{0}$ and $\bar{f}_{1}$, where $\bar{f}_{0}$ and $\bar{f}_{1}$ are maps from $\bar{V}$, the closure of $V$ to $X$. If $\bar{H}(x, t) \neq x$ for all $x \in B d(V)$ and for all $t$, then

$$
I\left(f_{0}\right)=I\left(f_{1}\right) \text { where } f_{0}=\bar{f}_{0}\left|V, f_{1}=\bar{f}_{1}\right| V .
$$

Proof. Since $H=\left.\bar{H}\right|_{V \times I}$ is a homotopy from $f_{0}$ to $f_{1}$, it suffices to verify that the set $F=$ $\{x \in V \mid H(x, t)=x$ for some $t\}$ is compact. Let $\left\{x_{j}\right\}$ be a sequence in $F$ converging to $x \in \bar{V}=V \cup B d(V)$. There exists a subsequence $\left\{t_{j}\right\}$ of those $t^{\prime}$ s in $I$ such that $\bar{H}\left(x_{j}, t_{j}\right)=$ $x_{j}$. Since $I$ is compact, a subsequence of $\left\{t_{j}\right\}$ converges to a point $t \in I$. By the continuity of $\bar{H}$, we have $\bar{H}(x, t)=x$. On the other hand, we know that $\bar{H}(x, t) \neq x$ for all $x \in B d(V)$; thus, $x \in V$ and $H(x, t)=x$. Consequently, $x \in F$. Therefore, $F$ is a closed subset of a compact space, hence $F$ is compact. This proves the proposition.

\section{The main formula}

Consider a compact topological manifold $M$ with boundary $\partial M$. We attach a collar to $M$ and call the resulting manifold $M^{\prime}: M^{\prime}=M \cup \partial M \sim \partial M \times\{0\} \partial M \times[0,1]$. Let $f: M \rightarrow M^{\prime}$ be a map such that $f(x) \neq x$ for all $x \in \partial M$. Since $M$ is compact, the fixed point set $F(f)$ is a compact set contained in $\stackrel{\circ}{M}=M \backslash \partial M$. For such $f: M \rightarrow M^{\prime}$, we define the index of $f$, denoted by $I(f)$, to be the fixed point index of the map $\left.f\right|_{M}$ given in Section 1 .

For specificity, we define the retraction $r$ : let $r: M^{\prime} \rightarrow M$ be the retraction from $M^{\prime}$ to $M$ given by the formula,

$$
\begin{gathered}
r(m)=m \quad \text { for } m \in M \\
r(b, t)=(b, 0) \sim b \quad \text { for }(b, t) \in \partial M \times[0,1] .
\end{gathered}
$$

Now we can formulate the main result of the section.

Now, assume $r^{\prime}$ is any retraction from $M^{\prime}$ to $M$ such that $r^{\prime}$ maps the collar $\partial M \times[0,1]$ into the boundary $\partial M$. Then the following theorem is true.

Theorem 3.1. One has that

$$
I(f)+I\left(\left.r^{\prime} f\right|_{\partial_{-} M}\right)=L\left(r^{\prime} f\right) .
$$

Furthermore,

$$
\begin{aligned}
L(r f) & =L\left(r^{\prime} f\right), \\
I\left(\left.r f\right|_{\partial_{-} M}\right) & =I\left(\left.r^{\prime} f\right|_{\partial_{-} M}\right),
\end{aligned}
$$

where $r$ is the standard retraction defined above and where $L(r f)$ denotes the Lefschetz number of $r f: M \rightarrow M$ and $\partial_{-} M=\{x \in \partial M \mid f(x) \notin M\}$.

Proof. First, we prove the formula $I(f)+I\left(\left.r^{\prime} f\right|_{\partial \_}\right)=L\left(r^{\prime} f\right)$. Let $V_{1}=\{x \in M \mid f(x) \in$ $\stackrel{\circ}{M}\}$ and $V_{2}=\left\{x \in M \mid f(x) \in M^{\prime} \backslash M\right\}$, then $V_{1}$ and $V_{2}$ are disjoint open subsets of the 
manifold $M$ and $V_{1} \cup V_{2}$ contains all the fixed points of the map $r^{\prime} f$. Indeed, if $x \notin$ $\left(V_{1} \cup V_{2}\right)$, then $f(x) \in \partial M$, and hence $r^{\prime} f(x)=f(x) \neq x$. Proposition 2.8 implies the equation

$$
I\left(r^{\prime} f\right)=I\left(\left.r^{\prime} f\right|_{V_{1}}\right)+I\left(\left.r^{\prime} f\right|_{V_{2}}\right) .
$$

Since $r^{\prime} f$ is a self-map from $M$ to $M$, so

$$
I\left(r^{\prime} f\right)=L\left(r^{\prime} f\right)
$$

We have

$$
L\left(r^{\prime} f\right)=I\left(\left.r^{\prime} f\right|_{V_{1}}\right)+I\left(\left.r^{\prime} f\right|_{V_{2}}\right) .
$$

Now, since $\left.r^{\prime} f\right|_{V_{1}}=\left.f\right|_{V_{1}}$ and $F(f) \subseteq V_{1}$, then

$$
I\left(\left.r^{\prime} f\right|_{V_{1}}\right)=I\left(\left.f\right|_{V_{1}}\right)=I(f) .
$$

Let us decompose the map $\left.r^{\prime} f\right|_{V_{2}}$ :

$$
\left.r^{\prime} f\right|_{V_{2}}: V_{2} \stackrel{\left.f\right|_{V_{2}}}{\longrightarrow} \partial M \times[0,1] \stackrel{r^{\prime}}{\longrightarrow} \partial M \stackrel{i}{\longrightarrow} M .
$$

The Commutativity 2.6 implies that

$$
I\left(\left.r^{\prime} f\right|_{V_{2}}\right)=I\left(\left.i r^{\prime} f\right|_{V_{2}}\right)=I\left(\left.r^{\prime} f i\right|_{i^{-1}\left(V_{2}\right)}\right)=I\left(\left.r^{\prime} f\right|_{\partial \_}\right) .
$$

Combining (3.6), (3.7), and (3.9), we obtain

$$
I(f)+I\left(\left.r^{\prime} f\right|_{\partial \_}\right)=L\left(r^{\prime} f\right) .
$$

This completes the proof of the formula holding for any retraction $r^{\prime}$. The following two lemmas will show that the terms in (3.10) are the same no matter which retraction $r^{\prime}$ is chosen.

LEMMA 3.2. The retraction $r$ is homotopic to $r^{\prime}$.

Proof. Consider the homotopy $H_{t}: M^{\prime} \rightarrow M, 0 \leq t \leq 1$, defined as follows:

$$
\begin{gathered}
H_{t}(m)=m \quad \text { for } m \in M, \\
H_{t}(b, s)=r^{\prime}(b, s t) \quad \text { for }(b, s) \in \partial M \times[0,1] .
\end{gathered}
$$

Clearly, $H_{0}=r$ and $H_{1}=r^{\prime}$. So, $r f$ and $r^{\prime} f$ are homotopic.

Lemma 3.3. $L\left(r^{\prime} f\right)=L(r f)$ and $I\left(\left.r f\right|_{\partial_{-} M}\right)=I\left(\left.r^{\prime} f\right|_{\partial_{-} M}\right)$.

Proof. By Lemma 3.2, $r f$ and $r^{\prime} f$ are homotopic and, consequently,

$$
L(r f)=L\left(r^{\prime} f\right)
$$

since the Lefschetz number $L$ is a homotopy invariant. 
6 Fixed point indices and manifolds with collars

Equations (3.10) and (3.12) with $r$ replacing $r^{\prime}$ imply that

$$
I\left(\left.r f\right|_{\partial_{-} M}\right)=I\left(\left.r^{\prime} f\right|_{\partial_{-} M}\right)
$$

This concludes the proof of Theorem 3.1.

Corollary 3.4. If $f: M \rightarrow M^{\prime}$ is a map such that $f(x) \notin M$ for any $x \in \partial M$, then $I(f)=$ $L(r f)-L\left(\left.r f\right|_{\partial M}\right)$.

Corollary 3.5. If $f: M \rightarrow M^{\prime}$ is without fixed points on the boundary $\partial M$ and $f(\partial M) \subset$ $M$, then $I(f)=L(r f)$.

Example 3.6. Consider a map $f: D^{n} \rightarrow \mathbb{R}^{n}$. Here $D^{n}$ is the unit ball and $S^{n-1}$ is the unit boundary sphere, so we can think of $\mathbb{R}^{n}$ as $D^{n}$ with an open collar attached.

(i) If $f\left(S^{n-1}\right) \subset D^{n}$, then $f$ has a fixed point.

(ii) If $f\left(S^{n-1}\right) \subset \mathbb{R}^{n} \backslash D^{n}$, then Corollary 3.4 implies that $I(f)=L(r f)-L\left(\left.r f\right|_{S^{n-1}}\right)=$ $1-\left(1+(-1)^{n-1} \operatorname{deg}\left(\left.r f\right|_{S^{n-1}}\right)\right)=(-1)^{n} \operatorname{deg}\left(\left.r f\right|_{S^{n-1}}\right)$.

Corollary 3.7. If $f: M \rightarrow M^{\prime}$ is homotopic to the inclusion map $M \hookrightarrow M^{\prime}$, then $I(f)+$ $I\left(\left.r f\right|_{\partial \_}\right)=\chi(M)$, where $\chi(M)$ denotes the Euler characteristic of $M$.

Proof. If $f: M \rightarrow M^{\prime}$ is homotopic to the inclusion map $M \hookrightarrow M^{\prime}$, then the composite map $r f: M \rightarrow M$ is homotopic to the identity map. Therefore $L(r f)=L(I d)=\chi(M)$.

Remark 3.8. Here is a more geometric proof of the main theorem (Theorem 3.1).

Proof. Let $D M$ be the double of $M$, that is, the union of two copies of $M$ intersecting on their boundaries. Let $R: D M \rightarrow M$ be the retraction which takes the second copy onto the first. Now $f \circ R: D M \rightarrow M$. Then the Lefschetz numbers $L(f)=L(f \circ R)$ since $R$ is a retraction, which splits the homology of $D M$, so that the traces of the induced map must be calculated only on the first copy $M$ of $D M$.

Also we consider $M \subset M^{\prime} \subset D M$. Then $R$ restricted to $M^{\prime}$ is equal to $r$. Now the fixed point set of $f \circ R$ consists of the fixed point set of $f$, in the interior of $M$, and the fixed point set $F(f \circ R)=F(f \circ r)$ contained in $\partial M$. Now the index of $r \circ f$ calculated on the open set $\partial_{M}$ is equal to the index calculated on a small open set $V$ of $M^{\prime}$ containing $\partial \_M$ which follows from the next lemma.

Lemma 3.9. One has that

$$
I\left(\left.r \circ f\right|_{\partial_{-} M}\right)=I\left(\left.r \circ f\right|_{V}\right) .
$$

Proof. Commutativity 2.6 implies that

$$
I\left(\left.r \circ f\right|_{V}\right)=I\left(\left.f \circ r\right|_{r^{-1}(V)}\right) .
$$

It is easy to see that the fixed point set of the map $\left.f \circ r\right|_{r^{-1}(V)}$ is $\{(b, t) \in \partial M \times(0$, 1] $\mid f(b)=(b, t)\}$ and the fixed point set of the map $\left.r f\right|_{V}$ is $\{b \in \partial M \mid f(b)=(b, t)$ for some $t\}$. 
We now define a homotopy $G_{s}, 0 \leq s \leq 1$, as the composite of the following maps

$$
\overline{\partial \_} \times I \stackrel{r}{\longrightarrow} \overline{\partial \_} M \stackrel{f}{\longrightarrow} \partial M \times I \stackrel{H_{s}}{\longrightarrow} \partial M \times I,
$$

where the map $H_{s}$ is defined as follows:

$$
H_{s}(b, t)=(b, s \bar{t}+(1-s) t), \quad \text { where } \bar{t} \text { is a constant, } 0<\bar{t} \leq 1 .
$$

Since the map $H_{0}=$ Identity, we have

$$
\begin{gathered}
G_{0}(x, t)=H_{0}(f r(x, t))=f r(x, t), \\
G_{1}(x, t)=H_{1}(f r(x, t))=(r f \times g)(x, t),
\end{gathered}
$$

where $r \circ f$ is a map from $\overline{\partial \perp}$ to $\partial M$ and $g: I \rightarrow I, g(t)=\bar{t}$, is the constant map. Furthermore, the restriction $\left.G_{s}\right|_{B d\left(\partial \_M \times I\right)}$ has no fixed points for any $0 \leq s \leq 1$. To see this, we look at a point $x \in B d\left(\partial \_M\right)$. We know then that $f(x) \in \partial M$ and $r f(x)=f(x) \neq x$, therefore, $G_{s}(x, t)=H_{s}(f r(x, t))=H_{s}(f(x))=H_{s}(f(x), 0)=(f(x), s \bar{t}) \neq(x, t)$.

Now the Axioms 2.9, 2.5, and 2.3 imply that

$$
\begin{gathered}
I\left(\left.f r\right|_{\partial \_M \times(0,1]}\right)=I\left(\left.r f\right|_{\partial \_M}\right) \cdot I(g)=I\left(\left.r f\right|_{\partial \_}\right) \cdot 1, \\
I\left(\left.r f\right|_{V}\right)=I\left(\left.f r\right|_{r^{-1}(V)}\right)=I\left(\left.f r\right|_{\partial_{-} M \times(0,1]}\right) .
\end{gathered}
$$

The last equality holds because $\partial \_M \times(0,1]$ contains the fixed point set of $\left(\left.f r\right|_{r^{-1}(V)}\right)$. Thus, $I\left(\left.r f\right|_{V}\right)=I\left(\left.r f\right|_{\partial M}\right)$.

Proof of Theorem 3.1. Consider the composite $M \stackrel{f}{\rightarrow} M^{\prime} \stackrel{r}{\rightarrow} M$. Let $V$ be the open set as in Lemma 3.3, then $V$ and $\stackrel{\circ}{M}$ are two open subsets of $M$ such that $V \cup \stackrel{\circ}{M}=M$. Clearly, $F(r f) \cap \stackrel{\circ}{M}$ and $F(r f) \cap V$ are disjoint. Using Additivity 2.2 and Normalization 2.4 of the fixed point indices, we have

$$
I\left(\left.r f\right|_{M}\right)+I\left(\left.r f\right|_{V}\right)=I(r f)=L(r f)
$$

Lemmas 3.2 and 3.3 then imply the equation

$$
I(f)+I\left(\left.r f\right|_{\partial_{-} M}\right)=L(r f) .
$$

\section{References}

[1] J. C. Becker and D. H. Gottlieb, Vector fields and transfers, Manuscripta Mathematica 72 (1991), no. 2, 111-130.

[2] C.-F. Benjamin, Fixed point indices, transfers and path fields, Ph.D. thesis, Purdue University, Indiana, 1990.

[3] R. F. Brown, Path fields on manifolds, Transactions of the American Mathematical Society 118 (1965), 180-191.

[4] , The Lefschetz Fixed Point Theorem, Scott, Foresman, Illinois, 1971. 
[5] A. Dold, Fixed point index and fixed point theorem for Euclidean neighborhood retracts, Topology. An International Journal of Mathematics 4 (1965), 1-8.

[6] L__ Lectures on Algebraic Topology, Die Grundlehren der mathematischen Wissenschaften, vol. 200, Springer, New York, 1972.

[7] _ The fixed point transfer of fibre-preserving maps, Mathematische Zeitschrift 148 (1976), no. 3, 215-244.

[8] E. Fadell, Generalized normal bundles for locally-flat imbeddings, Transactions of the American Mathematical Society 114 (1965), 488-513.

[9] D. H. Gottlieb, A de Moivre like formula for fixed point theory, Fixed Point Theory and Its Applications (Berkeley, CA, 1986) (R. F. Brown, ed.), Contemp. Math., vol. 72, American Mathematical Society, Rhode Island, 1988, pp. 99-105.

[10] _ A de Moivre formula for fixed point theory, ATAS de $5^{\circ}$ Encontro Brasiliero de Topologia 53 (1988), 59-67, Universidade de Sao Paulo, Sao Carlos S. P., Brasil.

[11] _ On the index of pullback vector fields, Differential Topology (Siegen, 1987) (U. Koschorke, ed.), Lecture Notes in Math., vol. 1350, Springer, Berlin, 1988, pp. 167-170.

[12] Z Zeroes of pullback vector fields and fixed point theory for bodies, Algebraic Topology (Evanston, IL, 1988), Contemp. Math., vol. 96, American Mathematical Society, Rhode Island, 1989, pp. 163-180.

[13] H. Hopf, Abbildungsklassen n-dimensionaler Mannigfaltigkeiten, Mathematische Annalen 96 (1927), no. 1, 209-224 (German).

[14] S. T. Hu, Fibrings of enveloping spaces, Proceedings of the London Mathematical Society. Third Series 11 (1961), 691-707.

[15] M. Morse, Singular points of vector fields under general boundary conditions, American Journal of Mathematics 51 (1929), no. 2, 165-178.

[16] J. Nash, A path space and the Stiefel-Whitney classes, Proceedings of the National Academy of Sciences of the United States of America 41 (1955), 320-321.

[17] C. C. Pugh, A generalized Poincaré index formula, Topology. An International Journal of Mathematics 7 (1968), 217-226.

Chen-Farng Benjamin: 705 Sugar Hill Drive, West Lafayette, IN 47906, USA

E-mail address: chenflben@gmail.com

Daniel Henry Gottlieb: Mathematics Department, Purdue University, West Lafayette, IN 47907, USA

E-mail address: gottlieb@math.ucla.edu 